\title{
Acute Kidney Injury with Levetiracetam in Patient with Epilepsy: A Case Report
}

\author{
Khalid Al-Quliti1, Rakan Maher Alhujeily² \\ ${ }^{1}$ Neurology Division, Department of Medicine, College of Medicine, Taibah University, Al-Madinah Almunwwarah, Saudi Arabia \\ ${ }^{2}$ College of Medicine, Taibah University, Al-Madinah Almunwwarah, Saudi Arabia \\ Email:kquliti@taibahu.edu.sa, Rakanma97@gmail.com
}

How to cite this paper: Al-Quliti, K. and Alhujeily, R.M. (2022) Acute Kidney Injury with Levetiracetam in Patient with Epilepsy: A Case Report. Case Reports in Clinical Medicine, 11, 48-54.

https://doi.org/10.4236/crcm.2022.113008

Received: February 6, 2022

Accepted: March 5, 2022

Published: March 8, 2022

Copyright ( 2022 by author(s) and Scientific Research Publishing Inc. This work is licensed under the Creative Commons Attribution International License (CC BY 4.0).

http://creativecommons.org/licenses/by/4.0/ (c) (i) Open Access

\begin{abstract}
Epilepsy is a common neurological disorder in neurology clinic. Levetiracetam is considered as one of common antiepileptic drugs used to manage epilepsy with good efficacy and tolerability profile. It is renally excreted and not depending on the cytochrome p450. It has adverse effects reported as somnolence, headaches, dizziness, depression and anxiety. Also, it was reported that levetiracetam can cause Acute kidney injury (AKI), renal profile disturbance, that may be related to its way of excretion and possible nephrotoxicity especially with high loading dose. We are reporting a young female patient with epilepsy presented to hospital with status epileptcus and started on loading dose of levetiracetam 3 grams and then maintenance dose of 1 gram twice daily seizure were controlled but she developed acute kidney injury that improved after discontinue leveriracetam and medical management without renal dialysis and discharged home in stable condition. Physician and health care providers should be aware of such rare adverse reaction and available management options for better patient care and outcome.
\end{abstract}

\section{Keywords}

Acute Kidney Injury, Epilepsy, Levetiracetam

\section{Introduction}

Epilepsy is a common neurological disorder, it is ranks fourth in the world's neurological disorders burden with lifetime prevalence of $6-7$ per 1000 people [1]. It is affecting all age group and both gender and requiring long-term, sometimes lifelong, treatment. Antiepileptic drugs (AEDs) are the main treatment option for epilepsy patients, and two-thirds of epileptic seizures can be controlled by AEDs. Levetiracetam (LEV) is relatively new AEDs which were approved as 
an adjunctive therapy for adults with focal epilepsy since 1999 in the US. It was approved in 2006 as monotherapy for adults and adolescents above 16 years of age with newly diagnosed focal-onset seizures with or without secondary generalization in Europe [1] [2]. Levetiracetam is considered as one of the most common antiepileptic drugs used to manage epilepsy. It is important to know that the way of elimination of levetiracetam is mainly occurs by renal excretion, and because of that, the mechanism of action is not depending on the cytochrome p450 [3] [4]. Levetirracitam can be administered intravenously or orally and it is effective medication and considered as a good option to be used in treatment of status epileptcus which is a prolonged or repeated attacks of seizures without regaining of conciseness. The most common adverse reaction of levetiracetam is asthenia, headaches, dizziness, somnolence, and behavioral changes [5] [6] [7]. Recent studies reported that there are some patients who have seizures and not known to have renal issues can have acute kidney injury when using levetiracetam, and surprisingly they are getting much better when they stopped take levetiracetam and using other antiseizure medication to control their seizures [8] [9] [10]. We are reporting a case of acute kidney injury induced by levetiracetam for a patient with history of epilepsy who presented to emergency room with status epilepticus.

\section{Case Presentation}

A 34-year-old female patient known to have epilepsy for 12 years she was carbamazepine $400 \mathrm{mg}$ orally twice daily with history of poor compliance to her medication brought to emergency department with status epilepticus as she had tonic-clonic seizures four times without regaining her consciousness. Vital signs showed a blood pressure (BP) of $137 / 86 \mathrm{mmHg}$, heart rate (HR) of 96 beats per minute (bpm), and Weight of $86 \mathrm{~kg}$, height of $169 \mathrm{~cm}$, her BMI was 30. Neurological examination she was conscious and drowsy with equal pupils and reactive to light, intact cranial nerve examination, and no signs of meningeal irritation, with normal motor and sensory examination.

\subsection{Investigation}

Her laboratory investigation result with normal reference (Table 1), Patient had brain Computer topography (CT) which was unremarkable and negative for acute pathology later Brain magnetic resonance imaging (MRI) was done and was unremarkable.

\subsection{Treatment and Course in Hospital}

Patient received lorazepam $2 \mathrm{mg}$ IV two doses separate to stop seizures then was started on intravenous levetiracetam 3 grams as loading dose diluted in at $100 \mathrm{ml}$ of a normal saline and administered over 20-minute intravenous infusion and was continued with levetiracetam $1000 \mathrm{mg}$ intravenously every 12 hours and was admitted to intensive care unit, Seizure was controlled with no recurrence; however, urine output was decrease and the patient developed oliguria. The patient 
Table 1. Laboratory investigation result on admission with normal reference.

\begin{tabular}{|c|c|}
\hline Test & Result (Normal Reference) \\
\hline White Blood Cell Count & $13.90\left(4.10-10.10 \times 10^{3} / \mathrm{uL}\right)$ \\
\hline Hemoglobin & $12.1(12.9-16.7 \mathrm{~g} / \mathrm{dL})$ \\
\hline Neutrophils & $16.2\left(1.40-6.80 \times 10^{3} / \mathrm{uL}\right.$ \\
\hline Lymphocytes & $2.5\left(1.10-2.90 \times 10^{3} / \mathrm{uL}\right)$ \\
\hline Monocytes & $0.9\left(0.20-1.00 \times 10^{3} / \mathrm{uL}\right)$ \\
\hline Platelets & $302\left(153-328 \times 10^{3} / \mathrm{uL}\right)$ \\
\hline Serum Glucose & $125(74-106 \mathrm{mg} / \mathrm{dL})$ \\
\hline Serum Blood Urea Nitrogen (BUN) & $14(9.0-20.0 \mathrm{mg} / \mathrm{dL})$ \\
\hline Serum Creatinine & $1.1(0.66-1.25 \mathrm{mg} / \mathrm{dL})$ \\
\hline Serum Sodium & $141(133-145 \mathrm{mEq} / \mathrm{L})$ \\
\hline Serum Potassium & $4.2(3.5-5.1 \mathrm{mEq} / \mathrm{L})$ \\
\hline Serum Chloride & $102(98-107 \mathrm{mEq} / \mathrm{L})$ \\
\hline Serum Calcium & $9.6(8.4-10.2 \mathrm{mg} / \mathrm{dL})$ \\
\hline Serum Total Protein & $9.2(6.3-8.2 \mathrm{~g} / \mathrm{dL})$ \\
\hline Serum Albumin & $4.6(3.5-5.0 \mathrm{~g} / \mathrm{dL})$ \\
\hline Serum Total bilirubin & $1.1(0.2-1.3 \mathrm{mg} / \mathrm{dL})$ \\
\hline Alanine transaminase (ALT) & $56(21-72 \mathrm{U} / \mathrm{L})$ \\
\hline Aspartate transaminase (AST) & $45(17-59 \mathrm{U} / \mathrm{L})$ \\
\hline Serum Lactate & $6.2(0.70-2.10 \mathrm{mmol} / \mathrm{L})$ \\
\hline Creatinine Kinase (CK) & $467(55-170)$ \\
\hline Urine protein & $42(5.0-11.0 \mathrm{mg} / \mathrm{dL})$ \\
\hline Urine sodium & $93(30.0-90.0 \mathrm{mEq} / \mathrm{L})$ \\
\hline Urine urea nitrogen & $62 \mathrm{mg} / \mathrm{dL}$ \\
\hline Urine creatinine & $124 \mathrm{mg} / \mathrm{dL}$ \\
\hline
\end{tabular}

was assessed by a neurology and nephrology teams and urinalysis showed large blood, and 1.010 of specific gravity, and increased urine sodium $(96 \mathrm{mEq} / \mathrm{L})$. No signs of hydronephrosis showed on abdominal ultrasound. Patient had no history of renal disease, intravenous contrast, or nephrotoxic medication. levetiracetam was discontinued after 4 days as a possible cause of AKI and received intravenous furosemide, 1/2 normal saline, and it was interchangeable with 5\% dextrose in water for volume expansion then urine output was improved gradually with follow up of Creatinine, BUN and CK levels continued to improve (Table 2) till normalized without requiring renal dialysis. Later lamotrigine 50 $\mathrm{mg}$, and to be taken twice daily was added to avoid seizure recurrence with no side effect, patient has no recurrence of seizure and renal function was normal till discharge from hospital after 20 dayd of admission with a follow up at outpatient neurology clinic after 2 month where she continue to have controlled seizures and normal renal function test. 
Table 2. Trend of laboratory result during admission.

\begin{tabular}{cccc}
\hline Day in hospital & $\begin{array}{c}\text { Creatinine (mg/dL) } \\
\text { (Reference: } \\
\text { 0.66 - 1.25 } \mathbf{~ g / d L ) ~}\end{array}$ & $\begin{array}{c}\text { BUN (mg/dL) } \\
\text { (Reference: } \\
\mathbf{9 . 0}-\mathbf{2 0 . 0} \mathbf{~ m g / d L )}\end{array}$ & $\begin{array}{c}\text { CK } \\
\text { (Reference: } \\
\mathbf{5 5 - 1 7 0 ~ U / L ) ~}\end{array}$ \\
\hline On admission & 1.13 & 14 & 467 \\
Day 2 & 2.53 & 17 & 426 \\
Day 3 & 4.62 & 24 & 512 \\
Day 4 & 7.36 & 41 & 734 \\
Day 5 & 8.14 & 57 & 1270 \\
Day 6 & 7.78 & 62 & 1082 \\
Day 7 & 7.16 & 64 & 927 \\
Day 8 & 6.48 & 58 & 644 \\
Day 10 & 4.13 & 47 & 489 \\
Day 12 & 2.62 & 36 & 261 \\
Day 14 & 1.21 & 26 & 192 \\
Day 15 & 1.13 & 19 & 153 \\
\hline
\end{tabular}

BUN: Blood urea nitrogen, CK: creatinine kinase.

\section{Discussion}

Levetiracetam is considered as a new and favorable antiepileptic drug because it is well-tolerated drug for many types of seizures and has minimal side effects. With generally good efficacy and safety profile [11] [12]. Due to its way of excretion, some renal adverse effects can occur, and rarely acute kidney injury can be caused by levetiracetam. In this report, we demonstrate the clinical and biomedical profile of a patient with relation between acute kidney injury and levetiracetam as an inducer, especially with high loading dose.

Our patient started to have decreased urine output and oliguria after taking levetiracetam for 4 days, and urinalysis showed large blood, 1.010 of specific gravity, and increased urine sodium $(96 \mathrm{mEq} / \mathrm{L})$, and the creatinine and $\mathrm{CK}$ were gradually increase and reached their highest level on the $5^{\text {th }}$ day, while BUN reached its highest level on the $7^{\text {th }}$ day. Levetiracetam was discontinued as it was thought to be the offending agent and all the creatinine, CK, and BUN were improved after the discontinuation of levetiracetam and later using lamotrigine 50 mg twice daily as an alternative antiepileptic medication to avoid seizure recurrence, as levetiracetam founded to be the likely cause in our case to induce acute kidney injury.

On reviewing the literature; Cases were reported that levetiracetam can cause AKI. One case about 26 years old male with history of epilepsy had tonic-clonic seizures for five times without regaining his consciousness. Prior his presentation, his medication at home was levetiracetam $750 \mathrm{mg}$ but he has poor compliance. He received $10 \mathrm{mg}$ of midazolam intramuscularly with the ambulance, then after he was arrived at ED, $4 \mathrm{mg}$ of midazolam was given to him to stop his 
seizures. The patient then noticed by the ED that he is in a status epilepticus phase, and after intubation, 4 grams loading dose of levetiracetam was given intravenously, then he was continued on levetiracetam $1000 \mathrm{mg}$. His initial laboratory investigations were elevated levels of lactate, creatinine, creatinine kinase, and BUN. levetiracetam discontinued, and $500 \mathrm{mg}$ of valproic acid was given to control seizures. The creatinine level returned to the baseline level after 20 days [13] [14]. In this patient he was young and presented with status epileptics similar to our patient. Whoever, the loading dose was higher compared to our patient and the duration of improvement of his renal function was longer than our patient.

Another case for 23 years old female presented with two episodes of generalized tonic-clonic seizure lasting for 1 minute for each one without regaining her level of consciousness. She received lorazepam $2 \mathrm{mg}$ and levetiracetam 1 gram as loading dose. The initial laboratory investigations were all normal. Also, MRI and EEG were unremarkable. She was admitted under neurology observation, and she was given $500 \mathrm{mg}$ of levetiracetam on the next day. After that, the patient had raised creatinine level. After nephrology evaluation, they found that the most likely reason for elevated creatinine is levetiracetam. Urinalysis has 1+ blood, and abdominal ultrasound showed increased echogenicity of bilateral renal cortexes. For that reason, they discontinued levetiracetam, and they used phenytoin as an alternative and fluids. By the $4^{\text {th }}$ day in the hospital, the creatinine level was started to improve and back to its baseline [8] [15].

A third case reported about 45 years old male medically free was presented with dizziness and gait unsteadiness, and on neurological examination the patient had impaired tandem gait and increased deep tendon reflex with left sided brisk reflexes. A brain MRI was done for him and showed T1 hypointense lesion within the right thalamus. After taking biopsy, it showed low grade infiltrating astrocytoma. Before the surgery, the patient received levetiracetam $500 \mathrm{mg}$ BID for seizure prophylaxis. Therefore, his symptoms worsened to seizures and the levetiracetam was increased over 2 months period to $3000 \mathrm{mg} /$ day. After that, he was noted to have elevated creatinine level and fraction excretion of sodium (FENa) was $>1 \%[16]$.

Levetiracetam considered to be nephrotoxic in some patients with Granulomatous interstitial nephritis (GIN), which can lead to hemodialysis requiring acute renal failure, and by withdrawal of the medication, complete recovery can be made [17] [18].

Date suggest that It is important to consider the possibility even in rare occasions of renal function deterioration secondary to levetiracetam as one of the differential diagnoses for any unexplained acute kidney injury, mainly during the first few weeks of levetiracetam administration [19] [20].

\section{Conclusion}

Levetiracitame in an effective and well tolerated new antiepileptic medication with good safety profile. Due to the renal excretion of levetiracetam, in this case 
report we have a young female patient who develops an acute kidney injury after that was in relation after starting her on levetiracitam with improvement after medical treatment with intravenous fluid and discontinue the possible offending agent which was thought as levetiracetam, she respond well and discharged in stable condition with good control of seizure with other antiepileptic medication. It is important for physician and health care provider to close monitor all patients who started on new antiepileptic medication especially if patient required a loading dose for rare adverse reactions like nephrotoxicity and acute kidney injury for better patient care and clinical outcome.

\section{Consent}

Approval was obtained from relevant regulatory committee, and informed consent was taken.

\section{Conflicts of Interest}

The authors declare that the research was conducted in the absence of any commercial or financial relationships that could be construed as a potential conflict of interest.

\section{References}

[1] Yi, Z.M., Wen, C., Cai, T., Xu, L., Zhong, X.L., et al. (2018) Levetiracetam for Epilepsy: An Evidence Map of Efficacy, Safety and Economic Profiles. Neuropsychiatric Disease and Treatment, 15, 1-19. https://doi.org/10.2147/NDT.S181886

[2] Erdinc, B., Ghanta, S., Andreev, A., Elkholy, K.O. and Sahni, S. (2020) Acute Kidney Injury Caused by Levetiracetam in a Patient with Status Epilepticus. Cureus, 12, e8814. https://doi.org/10.7759/cureus.8814

[3] Berkovic, S.F., Knowlton, R.C., Leroy, R.F., Schiemann, J. and Falter, U. (2007) Placebo-Controlled Study of Levetiracetam in Idiopathic Generalized Epilepsy. Neurology, 69, 1751-1760. https://doi.org/10.1212/01.wnl.0000268699.34614.d3

[4] Bodalia, P.N., Grosso, A.M., Sofat, R., Macallister, R.J., Smeeth, L., Dhillon, S., et al. (2013) Comparative Efficacy and Tolerability of Anti-Epileptic Drugs for Refractory Focal Epilepsy: Systematic Review and Network Meta-Analysis Reveals the Need for Long Term Comparator Trials. British Journal of Clinical Pharmacology, 76, 649-667. https://doi.org/10.1111/bcp.12083

[5] Yau, K., Burneo, J.G., Jandoc, R., et al. (2019) Population-Based Study of Risk of AKI with Levetiracetam. Clinical Journal of the American Society of Nephrology, 14, 17-26. https://doi.org/10.2215/CJN.07490618

[6] Alberto, V., Giovanni, P., Fabiana, D., Valentina, F., Francesco, C. and Gaetano, Z. (2015) The Adverse Event Profile of Levetiracetam: A Meta-Analysis on Children and Adults. Seizure, 31, 49-55. https://doi.org/10.1016/j.seizure.2015.07.004

[7] Thomas, L., Mirza, M.M.F., Shaikh, N.A. and Ahmed, N. (2019) Rhabdomyolysis: A Rare Adverse Effect of Levetiracetam. BMJ Case Reports, 12, e230851. https://doi.org/10.1136/bcr-2019-230851

[8] Spengler, D.C., Montouris, G.D. and Hohler, A.D. (2014) Levetiracetam as a Possible Contributor to Acute Kidney Injury. Clinical Therapeutics, 36, 1303-1306.

https://doi.org/10.1016/j.clinthera.2014.06.002 
[9] Shahbaz, N., Khan, S.A., Younus, S.M., et al. (2017) Levetiracetam Induced Increase in Creatine Phosphokinase Levels. Journal of College of Physicians and Surgeons Pakistan, 27, S63-S64.

[10] Health Canada: Summary Safety Review_KEPPRA (Levetiracetam)—Assessing the Potential Risk of Acute Kidney Injury (Acute Renal Failure/Interstitial Nephritis). https://www.canada.ca/en/health-canada/services/drugs-health-products/medeffect-can ada/safety-reviews/summary-safety-review-keppra-levetiracetam-assessing-potential-ris k-kidney-injury-renal-failure.html

[11] Zaccara, G., Giovannelli, F., Bell, G.S. and Sander, J.W. (2014) Network Meta-Analyses of Antiepileptic Drug Efficacy and Tolerability in Drug-Resistant Focal Epilepsies: A Clinical Perspective. European Journal of Clinical Pharmacology, 70, 647-654. https://doi.org/10.1007/s00228-014-1669-y

[12] Schachter, S.C. (2018) Antiseizure Drugs: Mechanism of Action, Pharmacology, and Adverse Effects..

https://www.uptodate.com/contents/antiseizure-drugsmechanism-of-action-pharm acology-and-adverse-effects

[13] Carnovale, C., Gentili, M., Antoniazzi, S., et al. (2017) Levetiracetam-Induced Rhabdmyolysis: Analysis of Reports from FDA Adverse Event Reporting System Database. Muscle Nerve, 56, E176-E178. https://doi.org/10.1002/mus.25972

[14] Kellum, J.A., Lameire, N., Aspelin, P., Barsoum, R.S., Burdmann, E.A., Goldstein, S.L., Herzog, C.A., Joannidis, M., Kribben, A., Levey, A.S., MacLeod, A.M., Mehta, R.L., Murray, P.T., Naicker, S., Opal, S.M., Schaefer, F., Schetz, M. and Uchino, S. (2012) KDIGO Clinical Practice Guideline for Acute Kidney Injury. Kidney International Supplements, 2, 1-138.

[15] Nash, D.M., Przech, S., Wald, R. and O'Reilly, D. (2017) Systematic Review and Meta-Analysis of Renal Replacement Therapy Modalities for Acute Kidney Injury in the Intensive Care Unit. Journal of Critical Care, 41, 138-144.

https://doi.org/10.1016/j.jcrc.2017.05.002

[16] Mahta, A., Kim, R.Y. and Kesari, S. (2012) Levetiracetam-Induced Interstitial Nephritis in a Patient with Glioma. Journal of Clinical Neuroscience, 19, 177-178.

https://pubmed.ncbi.nlm.nih.gov/22071460 https://doi.org/10.1016/j.jocn.2011.08.007

[17] Chau, K., Yong, J., Ismail, K., Griffith, N., Liu, M. and Makris, A. (2012) Levetiracetam-Induced Severe Acute Granulomatous Interstitial Nephritis. Clinical Kidney Journal, 5, 234-236. https://doi.org/10.1093/ckj/sfs023

[18] Malkina, A. (2019) Acute Kidney Injury (AKI). https://www.merckmanuals.com/professional/genitourinary-disorders/acute-kidney -injury/acute-kidney-injury-aki

[19] Mena-Martin, F., Gutierrez-Garcia, A., Martin-Escudero, J. and Fernandez-Arconada, O. (2018) Acute Kidney Injury and Creatine Kinase Elevation after Beginning Treatment with Levetiracetam. European Neurological Review, 13, 113-115. https://doi.org/10.17925/ENR.2018.13.2.113

[20] Sohn, S.Y., Kim, J.G., Kim, D.H., et al. (2017) Repeated Occurrence of Hyperckemia after Levetiracetam Administration. EC Neurology, 8, 150-154. 